\title{
Duplicidad de la vena renal derecha en Didelphis aurita (Didelphimorphia: Didelphidae): reporte de un caso*
}

\section{Double right renal vein in Didelphis aurita (Didelphimorphia: Didelphidae): case report}

\author{
Shirley Viana Peçanha, ${ }^{* *}$ Raquel Batista Junger Carvalho, ${ }^{* * *}$ Carlos Augusto dos Santos-Souza, ${ }^{* * *}$ Luiza Eula Marques, ${ }^{* * * *}$ \\ Paulo de Souza Junior, ${ }^{* * * *}$ Marcelo Abidu-Figueiredo**
}

\begin{abstract}
Resumen
El Didelphis aurita, conocido como comadreja de orejas negras, es un marsupial con amplia distribución en la América del Sur, frecuente tanto en los ambientes silvestres como antrópicos debido a su gran plasticidad ecológica. Animales de esta especie son comúnmente atendidos en la medicina de animales silvestres y vienen siendo empleados como modelos experimentales alternativos a especies tradicionales. El objetivo de ese trabajo fue relatar la ocurrencia de un caso de vena renal doble en un espécimen adulto, macho, de $D$. aurita. Para eso, se ha disecado un cadáver fijado y conservado en solución de formaldehído $10 \%$, previamente inyectado con látex de color azul por la porción torácica de la arteria aorta para facilitar la disección. El espécimen poseía dos venas renales derechas, una vena craneal $(11,3 \mathrm{~mm})$ y otra caudal $(10,0 \mathrm{~mm})$ que drenaban distintamente para una vena cava caudal. La vena renal izquierda y las arterias renales eran únicas. Aunque la duplicidad de la vena renal es reportada en otras especies como perros, gatos y felideos silvestres, este es el primer relato en el género Didelphis. El conocimiento de las variaciones anatómicas vasculares es relevante para la correcta ejecución de procedimientos veterinarios y en la interpretación de hallazgos experimentales en relación con el sistema urogenital.
\end{abstract}

Palabras clave: Animales silvestres, Comadreja de Orejas Negras, Marsupialia, Sistema cardiovascular.

\begin{abstract}
Didelphis aurita, known as black eared oposum, is a marsupial widely distributed in South America, frequently seen in both wild and anthropogenic environments due to its great ecological plasticity. This species is commonly treated in wildlife veterinary services and has been used as alternative experimental models instead of conventional species. The aim of this report was to describe a case of double renal vein in a male, adult, specimen of D. aurita. In order to make the dissection easier, the cadaver was fixed and preserved in $10 \%$ formaldehyde solution, and previously injected with latex in blue color by the thoracic aorta to facilitate dissection. The specimen had two right renal veins, one cranial $(11.3 \mathrm{~mm})$ and other caudal $(10.0 \mathrm{~mm})$ that drained distinctly for caudal vena cava. The left renal vein and the renal arteries were single. Although double renal vein has been reported in other species, such as dogs, cats and wild felids, this is the first mention in the genus Didelphis. The knowledge of the vascular anatomical variations is relevant for the correct execution of veterinary procedures and the interpretation of experimental findings in urogenital system research.
\end{abstract}

Keywords: Black-eared oposum, Cardiovascular system, Marsupialia, Wild animals.

\section{Introducción}

Las comadrejas son marsupiales que pertenecen a la orden Didephimorphia, familia Didelphidade, genero Didelphis (Linnaeus, 1758). La mayor parte de las comadrejas del nuevo mundo tienen distribución geográfica que se extiende del sur de Canadá a porción central de Argentina, ocurriendo en una amplia variedad de hábitats, incluyendo áreas de montañas y florestas (Ventura et al., 2002). Fueron descriptas seis especies dentro del género: $D$. marsupialis (Linnaeus, 1758), $D$. virginiana (Kerr, 1792), D. aurita (Wied-Neuwied, 1826), D albiventris (Lund, 1840), D. pernigra (Allen, 1900), y D. imperfecta (Mondolfi e Pérez-Hernández., 1984) (Lemos e Cerqueira, 2002).

La especie Didelphis aurita, conocida como comadreja de orejas negras, se distribuye en la porción leste, centro oeste y sur de Brasil, Paraguay y Argentina (Cerqueira e Lemos, 2000). Debido

*Recebido em 28 de outubro de 2018 e aceito em 18 de março de 2019.

${ }^{* *}$ Departamento de Anatomia Animal e Humana/ICB/UFRRJ, Seropédica, RJ, Brasil.

***Parque Nacional da Serra dos Órgãos, Teresópolis, Rio de Janeiro, RJ, Brasil.

${ }^{* * * *}$ Centro de Ciências Biológicas e da Natureza/ UFAC, Rio Branco, AC - Brasil.

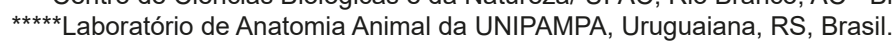

Autor para correspondência: Prof. Dr. Marcelo Abidu-Figueiredo - Departamento de Anatomia Animal e Humana da Universidade Federal Rural do Rio de Janeiro (UFRRJ). Rodovia BR-465, km 7, CEP 23890-000 Seropédica, RJ, Brasil. marceloabidu@gmail.com. 
a su gran plasticidad ecológica a los más diversos hábitats, se encuentran en poblaciones numerosas incluso en los grandes centros urbanos. Estos animales presentan dieta diversificada, la cual incluye residuos de alimentación humana, lo que aumenta su proximidad de áreas antrópicas (Albano et al., 2011).

Animales del género Didelphis viene siendo utilizados como especie alternativa no convencional para animales de laboratorios (D'Andrea et al. 2002), incluso en ensayos respecto a enfermedades del sistema urinario (Steinhardt et al., 1990; Liapis et al., 1997). A pesar de los avances en investigaciones básicas y aplicadas involucrando animales silvestres, se verifica que las informaciones referentes a las venas renales son escasas, especialmente mientras a sus variaciones numéricas y locales de drenaje.

En el caso de los mamíferos, las variaciones numéricas en las venas renales fueron caracterizadas en el perro (Reis e Tepe 1956), en el gato (Campos et al., 2014, Stocco et al., 2014), en el Leopardus guttulus (gato-del-mato-pequeño) (Stocco et al., 2018) y en el Leopardus pardalis (jaguatirica) (Stocco et al., 2017).

El presente estudio pretende describir la duplicidad de la vena renal derecha en un espécimen de Didelphis aurita como importante variación anatómica.

\section{Relato de caso}

Durante las actividades de disección realizadas en 23 cadáveres de Didelphis aurita en el Laboratorio de Enseñanza e Investigación en Morfología de los Animales Domésticos y Salvajes (LEPeMADS) del Departamento de Anatomía Animal y Humana, de la Universidad Federal Rural del Río de Janeiro (UFRRJ), Brasil, se observó una variación numérica de la vena renal derecha en un espécimen adulto macho. Todos los cadáveres fueron recogidos muertos en carreteras entorno del Parque Nacional de la Sierra de los Órganos, Teresópolis, Río de Janeiro, Brasil.

El cadáver tuvo su longitud rostro-sacral (desde la punta del hocino hasta la raíz de la cola) tallado con cinta métrica y posicionado en decúbito lateral derecho. Luego, el tórax fue abierto y disecado para revelación de la aorta torácica, en la cual fue introducida una sonda uretral número 2. De ese modo, el sistema arterial fue "lavado" con solución fisiológica de $\mathrm{NaCl}$ $0,9 \%$, y después fijado con solución de formaldehido $10 \%$. Después, fue inyectado por la sonda una solución acuosa (diluida 1:1) de Petrolatex S-65 (Refinería Duque de Caxias REDUC Petrobras, Duque de Caxias-RJ) mesclado al colorante azul (Suvinil $x a d r e z^{\circledR}$ ). Luego, el cadáver fue sumergido en una caja de polietileno conteniendo solución de formaldehido a 10\% para la finalización del proceso de fijación e polimerización del látex.

Transcurridos siete días de fijación, se procedió el lavaje en agua corriente, abertura y disecación de la cavidad peritoneal para exponer los riñones e sus respectivos vasos. Con un calibre digital (marca Eda ${ }^{\circledR}$, precisión $\pm 0,01 \mathrm{~mm}$ ) fueron obtenidas las medidas renales (longitud, anchura y espesura) y longitud de las venas renales por examinador único en duplicado.

\section{Discusión y conclusión}

El animal poseía $540 \mathrm{~mm}$ de longitud rostro-sacral, presentando el riñón derecho con 35,5 mm de longitud, 21,5 $\mathrm{mm}$ de anchura y $13,9 \mathrm{~mm}$ de espesura, ubicándose al nivel de la décima segunda vertebra torácica a la segunda vértebra lumbar (T12 a L2).

El espécimen presentó dos venas renales derechas, una craneal y otra caudal, ambas drenando independientemente para la vena cava caudal y sin recibir tributarias (Figura 1). La primera medió $11,3 \mathrm{~mm}$ de longitud y la segunda $10,0 \mathrm{~mm}$. Las dos venas se encontraban cranealmente a vena contralateral. La vena craneal emergió de la porción más dorsal del hilo, mientras la vena caudal de la porción ventral. Ambas se posicionaban ventralmente en relación a la única arteria renal derecha.

El riñón izquierdo midió $36,7 \mathrm{~mm}$ de longitud, 16,7 mm de anchura y $29,5 \mathrm{~mm}$ de espesura, y se localizó en el artejo correspondiente a las vértebras torácicas (T12 y T13) y lumbares ( L1 y L2). Presentó una sola vena renal con longitud de 12,4 mm.

Souza et al. (1992) estudiaron el drenaje venoso en 30 pares de riñones de Didelphis aurita, y no observaron duplicidad de la vena renal. En el presente estudio se observó en apenas un riñón, derecho, la formación de dos venas renales después de la disección de 23 especímenes. Juntando los hallazgos del presente estudio al de Souza et al. (1992), se puede aludir que se trata de una condición rara, presente en cerca de 1 a $2 \%$ de los individuos de esta especie.

En la especie humana, la ocurrencia de la vena renal doble varía entre 9 a 11\%, pudiendo ocurrir en cualquiera de los antimeros (Duques et al. 2002). Hassan et al. (2017) documentaron no solo venas renales dobles, como triplas y cuádruplas en cadáveres humanos.

En el estudio realizado por Reis y Tepes (1956) con 500 perros mestizos, 287 machos y 213 hembras, fue observado la presencia de vena renal derecha doble en apenas $1 \%$ ( $n$ = 5) y la vena renal izquierda se presentó única en todos los casos, que difieren de los resultados encontrados por Fagundes et al. (1990), cuya duplicidad fue encontrada solamente en el antimero izquierdo. En los gatos domésticos, Campos et al. (2014) y Stocco et al. (2014) relataron venas renales dobles en ambos los riñones y triplas solo en los riñones derechos, respectivamente.

La duplicidad de las venas renales no está restringida en las especies de animales domésticos. Esa variación anatómica fue descrita en Leopardus pardalis (Stocco et al. 2017) y Leopardus guttulus (Stocco et al. 2018). Santos-Sousa et al. (2016) observaron en un riñón izquierdo de un conejo que la vena renal no drenaba su contenido para la vena cava caudal y no mantuvo ninguna comunicación con la vena cava caudal, se anastamosando con la vena abdominal craneal.

La ocurrencia de variaciones numéricas en vasos renales es más frecuente en las arterias que en las venas, habiendo sido descritas en perros (Jain et al., 1985; Oliveira e Guimarães., 2007; Marques-Sampaio et al., 2007; Alonso e AbiduFigueiredo., 2008), gatos (Pestana et al., 2011) y conejos (Almeida et al., 2013). 
Debido a su alta concentración en áreas antrópicas, los animales del género Didelphis son comúnmente recoletos y llevados a las clínicas veterinarias y centros de rescate para los atendimientos (Albano et al., 2011; Santos et al., 2011; Rorato-Nascimento e Pinto., 2016). Las anomalías congénitas como agenesia del riñón derecho ya fueron descritas en el género Didelphis (Gupta e Feldman, 1975). Los estudios de fisiología de las glándulas adrenales que envolvían la canulación quirúrgica de la vena renal para la colecta de amuestras fueron realizadas por Beck et al. (1969) en animales del mismo género. Por lo tanto, el conocimiento del padrón anatómico y posibles variaciones, es importante para la ejecución y interpretación de los hallazgos experimentales y para la práctica médica veterinaria, contribuyendo para la mejora de los distintos protocolos clínicos y quirúrgicos en esta especie.

Así, la divulgación de los hallazgos de las variaciones anatómicas en estas especies es importante para la práctica médica veterinaria y contribuye para al perfeccionamiento de diferentes protocolos clínicos y quirúrgicos.

Por lo tanto, se deben considerar las variaciones numéricas de las venas renales en la ejecución de los procedimientos quirúrgicos, radiológicos y experimentales en estas especies, con propósito de evitar errores que sean cometidos por falta de conocimiento de la posibilidad de existencia de venas renales dobles.

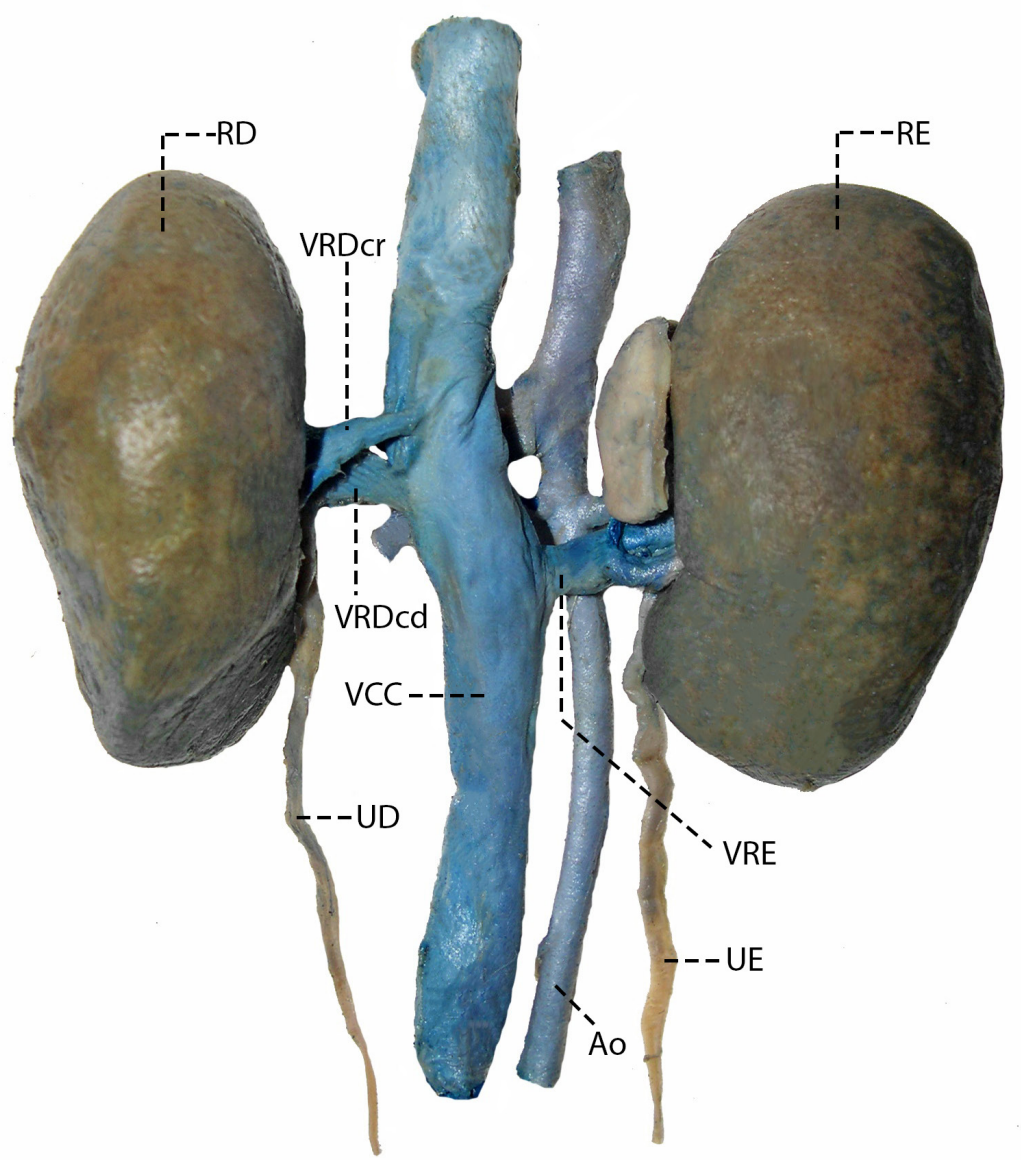

Figura 1: Fotomacrografía con vista ventral de los riñones, uréteres y vasos correlacionados de un espécimen adulto, macho, de Didelphis aurita. Arteria aorta abdominal (AO), riñón derecho (RD), riñón izquierdo (RE), uréter izquierdo (UE), uréter derecho (UD), vena renal izquierda (VRE), vena renal derecha caudal (VRDcd), vena renal derecha craneal (VRDcr), vena cava caudal (VCC). Barra de escala: $10 \mathrm{~mm}$

\section{Agradecimientos}

Al Consejo Nacional de Desarrollo Científico y Tecnológico (CNPq), por la subvención concedida a la primera autora.

Al Programa de Desarrollo Académico de UNIPAMPA por la subvención concedida a la cuarta autora.

Al Coordinación de Perfeccionamiento de Personal de Nivel Superior - Brasil (CAPES) - Código de Financiamiento 001; FAPERJ e CNPq. 


\section{Referencias}

ALBANO, A.P.N.; MENDES, J.F.; COIMBRA, M.A.A.; MEIRELES, M.C.A.; Ocorrência de Malassezia pachydermatis em gambá de orelha branca (Didelphis albiventris) - relato de caso. Clín. Vet., v.91, n.1, p.32-44, 2011.

ALMEIDA, B.B.; BARRETO, U.H.; COSTA, O.M.; ABIDUFIGUEIREDO, M. Double renal artery in rabbits. Biosci. J., v.29, n.1, p.1294-1295, 2013.

ALONSO, L.S.; ABIDU-FIGUEIREDO, M. Artéria renal dupla originando da aorta em cão: relato de caso. Semin. Cien. Agrar, v.29, n.1, p.185-188, 2008.

BECK, R.R.; BROWNELL, K.A.; BESCH, P.K. A further study of adrenal function in the opossum (Didelphis virginiana). Gen. Comp. Endocrinol, v.13, n.2, p.165-172, 1969.

CAMPOS, C.B.A.; ROCHA, P.S.; ABIDU-FIGUEIREDO, M. Veia renal dupla em gatos: relato de casos. Rev. Acad. Ciên. Anim. v.12, n.1, p.127-131, 2014.

CERQUEIRA, E.; LEMOS, B. Morphometric differentiation between Neotropical black-eared opossums, Didelphis marsupialis and $D$. aurita (Didelphimorphia, Didelphidae). Mammalia, v.64, n.1, p.319-327, 2000.

D'ANDREA, P.S.; ROQUE, A.L.R.; TEIXEIRA, B.R. Alternativas para Animais de Laboratório: uso de animais não-convencionais - roedores silvestres. In: ANDRADE, A.; PINTO, S.C.; OLIVEIRA, R.S. orgs. Animais de Laboratório: criação e experimentação. Rio de Janeiro, Fiocruz, 2002, p. 353-360.

DUQUES, P.; RODRIGUES, J.R.; SILVA, N.F.B.; ELRY, M.V.S.; TOLÊDO, E.S. Estudo anatômico da veia renal esquerda de cadáveres humanos brasileiros. Medicina, v.35, n.2, p.184-191, 2002.

FAGUNDES, G.M.; SOUZA, A.; BORELLI, V.; RIELLA, A.C.M. Contribuição ao estudo da drenagem sanguínea do rim de cães (Canis familiaris - Linnaeus, 1758). Biotemas, v.3, n.1, p.117-127, 1990.

GUPTA, B.N.; FELDMAN, D.B. Renal agenesis in guinea pig and opossum. Lab. Anim. Sci, v.25, n.2, p.238-40. 1975.

HASSAN, S.S.; EL-SHAARAWY, E.A.; JOHNSON, J.C.; YOUAKIM, M.F.; ETTARH, R. Incidence of variations in human cadaveric renal vessels. Folia Morphol. v.10, n.1, p.1644-3284, 2017.

JAIN RK, DHINGRA LD, KUMAR S, SHARMA DN.

Vascularization of kidneys in dogs (Canis familiaris). Indian J Anim Sci, v. 55, p. 406-409, 1985.

LEMOS, B; CERQUEIRA, R. Morphological differentiation in the white-eared opossum group (Didelphidae: Didelphis). J. Mammal, v.83, n.1, p.354-369, 2002.

LIAPIS, H.; VOGLER, G.; STEINHARDT, G.F. North American opossum Didelphis virginiana as a fetal nephrotoxicity model: Histologic and ultrastructural assessment of uranyl nitrate (UN) induced damage. Microsc. Res. Tech, v.39, n.1, p.285-296, 1997.
MARQUES-SAMPAIO, BPS., PEREIRA-SAMPAIO MA, HENRY RW, FAVORITO LA, AND SAMPAIO FJB. Dog kidney: anatomical relationships between intrarenal arteries and kidney collecting system. Anat. Rec. v. 290, p. 1017-1022, 2007.

OLIVEIRA, F.S.; GUIMARÃES, G.C. Duplicidade da artéria renal em cão. Ciência. Rural, v.37, n.6, p.1817 - 1819, 2007.

PESTANA, F.M.; ROZA, M.S.; HERNANDEZ, J.M.F.; SILVA, B.X.; ABIDU-FIGUEIREDO, M. Artéria renal dupla em gato: relato de caso. Semin. Cien. Agrar, v.32, n.1, p.325-330, 2011.

REIS, R.H.; TEPE, P. Variations in the pattern of renal vessels and their relation to the type posterior vena cava in the dog (Canis familiaris). Am. J. Anat. v. 99, n.1, p.1-15, 1956.

RORATO-NASCIMENTO, A.M.M.; PINTO, C.M. Levantamento dos atendimentos clínicos de mamíferos silvestres e exóticos no Setor de Clínica e Cirurgia de Animais Silvestres do Hospital Veterinário, Universidade de Santo Amaro, São Paulo, no período de Agosto de 2012 a Agosto de 2014. Anais do I COPESAH (Congresso de Pesquisa em Saúde Animal e Humana). ISSN 2358-4610. Universidade Estadual de Londrina. p.184-186, 2016.

SANTOS-SOUSA, C.A.; STOCCO, A.V.; FERREIRA, L.T.; SILVA, S.C.; GOMES, M.S.; ABIDU-FIGUEIREDO, M. Drenagem atípica da veia renal em coelho: Relato de caso. Braz. J. Vet. Med, v.38, n,1, p.105-107, 2016.

SANTOS, A.L.Q.; SILVA, J.M.M.; PEREIRA, H.C.; NASCIMENTO, L.R.; MENEZES, L.T.; KAMINISHI, Á.P.S. Tratamento de traumatismo craniano em gambá Didelphis albiventris Linnaeus, 1758 (Marsupialia, Didelphidae). PUBVET, v.5, n.17, p.1110, 2011.

SOUZA, A.; BORELLI, V.; FAGUNDES, G.M.; BRAGA, M.T.T. Contribuição ao estudo da drenagem sanguínea do rim de gambá (Didelphis aurita - Von Ihering, 1827). Biotemas, v.5, n.2, p.103114, 1992.

STEINHARDT, G.; SALINAS-MADRIGAL, L.; FARBER, R.; LYNCH, R.; VOGLER, G. Experimental Ureteral Obstruction in the Fetal Opossum. I. Renal Functional Assessment. J. Urol, v.144, n.2, p.564-566, 1990.

STOCCO, A.V.; STOCCO, N.V.; SANTOS-SOUZA, C.A.; ABIDUFIGUEIREDO, M. Veia renal tripla em gato: relato de casos. Rev. Port. Ciênc Vet, v.109, n.1, p.120-122, 2014.

STOCCO, A.V.; SILVA, S.C.; TOLEDO, K.S.; SOUSA, C.A.S.; CARVALHO, R.B.J.; ABIDU-FIGUEIREDO, M. Veia renal direita dupla em jaguatirica (Leopardus pardalis): relato de caso. Rev. Port. Ciênc. Vet. v.112, n.1, p.83-86, 2017.

STOCCO, A.V.; SILVA, S.C.; TOLEDO, K.S.; SOUSA, C.A.S.; CARVALHO, R.B.J.; ABIDU-FIGUEIREDO, M. Duplicidade da veia renal direita em gato-do-mato- pequeno (Leopardus guttulus): relato de caso. Rev. Acad. Ciênc. Anim, v.16, n.1, p.1-6, 2018.

VENTURA, J.; SALAZAR, M.; PÉREZ-HERNÁNDEZ, R.; LÓPEZ-FUNSTER, M.J. Morphometrics of the genus Didelphis (Didelphimorphia: Didelphidae) in Venezuela. J. Mammal, v.83, n.4, p.1087-1096, 2002 